\title{
Characterizing the Short-Term Habituation of Event-Related Evoked Potentials
}

\author{
(D)Flavia Mancini, ${ }^{1,2}$ Alessia Pepe, ${ }^{3}$ Alberto Bernacchia, ${ }^{2}$ Giulia Di Stefano, ${ }^{3}$ (D) André Mouraux, ${ }^{4}$ and \\ (D)Gian Domenico lannetti ${ }^{1,5}$
}

\section{DOI:http://dx.doi.org/10.1523/ENEURO.0014-18.2018}

\begin{abstract}
${ }^{1}$ Department of Neuroscience, Physiology and Pharmacology, University College London, London WC1E 6BT, United Kingdom, ${ }^{2}$ Computational and Biological Learning, Department of Engineering, University of Cambridge, Cambridge CB2 1PZ, United Kingdom, ${ }^{3}$ Department of Human Neuroscience, University of Rome "La Sapienza," Rome 00185, Italy, ${ }^{4}$ Institute of Neuroscience, Université Catholique de Louvain, Brussels B-1200, Belgium, and ${ }^{5}$ Neuroscience and Behaviour Laboratory, Istituto Italiano di Tecnologia (IIT), Rome, Italy
\end{abstract}

\begin{abstract}
Fast-rising sensory events evoke a series of functionally heterogeneous event-related potentials (ERPs). Stimulus repetition at $1 \mathrm{~Hz}$ induces a strong habituation of the largest ERP responses, the vertex waves (VWs). VWs are elicited by stimuli regardless of their modality, provided that they are salient and behaviorally relevant. In contrast, the effect of stimulus repetition on the earlier sensory components of ERPs has been less explored, and the few existing results are inconsistent. To characterize how the different ERP waves habituate over time, we recorded the responses elicited by 60 identical somatosensory stimuli (activating either non-nociceptive $A \beta$ or nociceptive A $\delta$ afferents), delivered at $1 \mathrm{~Hz}$ to healthy human participants. We show that the well-described spatiotemporal sequence of lateralized and vertex ERP components elicited by the first stimulus of the series is largely preserved in the smaller-amplitude, habituated response elicited by the last stimuli of the series. We also found that the earlier lateralized sensory wave habituates across the 60 trials following the same decay function of the VWs: this decay function is characterized by a large drop at the first stimulus repetition followed by smaller decreases at subsequent repetitions. Interestingly, the same decay functions described the habituation of ERPs elicited by repeated non-nociceptive and nociceptive stimuli. This study provides a neurophysiological characterization of the effect of prolonged and repeated stimulation on the main components of somatosensory ERPs. It also demonstrates that both lateralized waves and VWs are obligatory components of ERPs elicited by nonnociceptive and nociceptive stimuli.
\end{abstract}

Key words: EEG; ERP; habituation; nociception; somatosensory

\section{Significance Statement}

Our results provide a functional characterization of the decay of the different event-related potential (ERP) components when identical fast-rising somatosensory (nociceptive and non-nociceptive) stimuli are repeated at $1 \mathrm{~Hz}$. These stimuli elicit ERPs obligatory contributed by both early lateralized components and late vertex components, even when stimulus repetition minimizes stimulus relevance. This challenges the view that lateralized waves are not obligatorily elicited by nociceptive stimuli. Furthermore, the lateralized and vertex waves (VWs) habituate to stimulus repetition following similar decay functions, which are unlikely explained in terms of fatigue or adaptation of skin receptors.

Received January 9, 2018; accepted August 13, 2018; First published , 20.

The authors declare no competing financial interests.
Author contributions: F.M., A.M., and G.D.I. designed research; F.M., A.P., and G.D.S. performed research; F.M. contributed unpublished reagents/analytic tools; F.M., A.P., and A.B. analyzed data; F.M., A.B., and G.D.I. wrote the paper. 


\section{Introduction}

Sudden sensory events evoke a series of transient responses in the ongoing electrocortical activity [eventrelated potentials (ERPs)]. ERPs are functionally heterogeneous and reflect the activity of distinct cortical generators overlapping in time and space (Sutton et al., 1965). Since these generators include both sensory and associative cortical areas, the scalp distribution of the early lateralized ERP components elicited by stimuli of different modalities partly differs depending on the modality of the sensory input. In contrast, the scalp distribution of the late and largest ERP components is virtually identical regardless of the modality of the eliciting stimulus (Mouraux and lannetti, 2009): it consists in a biphasic negative-positive deflection widespread over the scalp and maximal at the vertex, often referred to as vertex wave (VW) or vertex potential (Bancaud et al., 1953).

The VW amplitude is maximal when fast-rising stimuli are presented using large and variable inter-stimulus intervals of several seconds (Mouraux and lannetti, 2009; Huang et al., 2013), or when the stimulus reflects behaviorally relevant changes within a regular series of otherwise identical stimuli (Snyder and Hillyard, 1976; Valentini et al., 2011; Ronga et al., 2013). In contrast, when identical stimuli are monotonously repeated at short and regular intervals (e.g., 0.5 or $1 \mathrm{~Hz}$ ), the VW amplitude strongly decays (Jasper and Sharpless, 1956; Ritter et al., 1968; Davis et al., 1972; Mouraux and lannetti, 2009; Liang et al., 2010; Wang et al., 2010). Although the decay of the VW due to repeated stimulation at different frequencies has been described (Fruhstorfer et al., 1970; Greffrath et al., 2007), a formal characterization of how the different constituent components of the ERP habituate over time is still missing. This is particularly important considering that previous studies suggested that neural activity in different cortical regions adapts to repeated stimulation at different timescales: for instance, neural activity in associative regions elicited by trains of innocuous, somatosensory stimuli decays faster than neural activity in sensory cortices (Forss et al., 2001; Venkatesan et al., 2014). However, these results may not generalize to responses elicited by noxious somatosensory stimuli: a previous study has suggested that the repetition of intraepidermal nociceptive stimuli at $1 \mathrm{~Hz}$ for $1 \mathrm{~min}$ fully suppresses lateralized evoked responses (Mouraux et al., 2013).

Therefore, our primary objective was to describe the short-term habituation of the different constituents of somatosensory nociceptive and non-nociceptive ERPs: both the large centrally-distributed VWs (N2 and P2

F.M. and G.D.I. were supported by the Wellcome Trust Strategic Award COLL JLARAXR. G.D.I. is additionally supported by a European Research Council (ERC) Consolidator Grant (PAINSTRAT) and by the Medical Research Council. A.M. is supported by an ERC Starting Grant (PROBING-PAIN).

Correspondence should be addressed to Flavia Mancini, at the above address, E-mail: fm456@cam.ac.uk.

DOI:http://dx.doi.org/10.1523/ENEURO.0014-18.2018

Copyright (C) 2018 Mancini et al.

This is an open-access article distributed under the terms of the Creative Commons Attribution 4.0 International license, which permits unrestricted use, distribution and reproduction in any medium provided that the original work is properly attributed. waves) and the smaller lateralized somatosensory waves (N1 and P4 waves). These are all the known waves elicited by nociceptive stimulation (Treede et al., 1988; Valentini et al., 2012; Hu et al., 2014). As in Mouraux et al. (2013), we recorded EEG while delivering trains of 60 identical stimuli at $1 \mathrm{~Hz}$. In one group of healthy participants, we transcutaneously and electrically stimulated nerve trunks, activating directly all large-diameter $A \beta$ somatosensory afferents and eliciting non-painful sensations. In a separate group of participants, we used radiant-heat stimuli that selectively activate skin nociceptors and elicit sensations of $A \delta$-mediated pinprick pain. We did not use intraepidermal electrical stimulation of nociceptive afferents (Mouraux et al., 2013), because it can induce strong habituation of peripheral nociceptors (the stimulus is delivered always in the same location, whereas radiant heat stimuli can be easily displaced to reduce nociceptor fatigue). The use of two different somatosensory stimuli allowed to cross-validate and generalize our findings across different sensory pathways.

We addressed two complementary questions. First, we statistically assessed whether the main response components were present in both the non-habituated ERP (i.e., the ERP elicited by the first stimulus of a series) and the habituated ERP (i.e., the ERP elicited by later stimuli that yield a stable, habituated response). The rationale for this decision was the consistent observation that the amplitude of the main ERP waves (i.e., VWs) decays only minimally after the first few stimulus repetitions (Ritter et al., 1968; Fruhstorfer et al., 1969, 1970; Fruhstorfer 1971; Greffrath et al., 2007; Mouraux et al., 2013), a finding corroborated by the present results (Figs. 1-4). Second, we asked whether and how the lateralized and VWs habituated throughout the block of 60 stimuli. We used singular value decomposition (SVD) to separate the ERP waveform from its amplitude change across stimulus repetitions. SVD provides a small number of components that best approximate the data and explain most of its variance (Golub and Reinsch, 1970). This approach allowed us to investigate the decay function of small ERP components, such as the lateralized waves.

\section{Materials and Methods}

\section{Participants}

Thirty-two healthy subjects (14 women) aged 19-31 years (mean \pm SD: $23.6 \pm 3.9$ ) participated in the study, after having given written informed consent. All experimental procedures were approved by the ethics committee of University College London (2492/001).

\section{Transcutaneous electrical stimulation of $\boldsymbol{A} \boldsymbol{\beta}$ fibers}

Innocuous stimulation of $A \beta$ afferents consisted of square-wave pulses (100- $\mu$ s duration), generated by a constant current stimulator (DS7A, Digitimer). Stimuli were delivered through a bipolar electrode placed above the superficial radial nerve and elicited a paresthetic sensation in the corresponding innervation territory. $A \beta$ detection thresholds were identified using the method of ascending staircases, on the right hand. The detection threshold was defined as the average of the lowest stim- 
ulus energy eliciting a sensation in three consecutive trials. Electrical stimuli were delivered at $\sim 300 \%$ of each individual's $A \beta$ detection threshold. Stimulus intensity was slightly adjusted to elicit sensations of comparable intensities on the left and right hands (mean \pm SD, $17.4 \pm$ $11.4 \mathrm{~mA}$ ) and to make sure that the elicited sensation was never painful.

\section{Cutaneous laser stimulation of $\mathbf{A} \boldsymbol{\delta}$ and $\mathbf{C}$ fibers}

Nociceptive stimuli were radiant heat pulses generated by an infrared neodymium:yttrium-aluminum-perovskite laser with a wavelength of $1.34 \mu \mathrm{m}$ (Nd:YAP; Electronical Engineering). At this wavelength, laser pulses excite $A \delta$ and $C$ nociceptive free nerve endings in the epidermis directly and selectively, i.e., without coactivating touchrelated $A \beta$ fibers in the dermis (Bromm and Treede, 1984; Baumgärtner et al., 2005; Mancini et al., 2014). The duration of each laser pulse was $4 \mathrm{~ms}$.

Laser stimuli were delivered within a squared skin area $(4 \times 4 \mathrm{~cm})$ centered on the dorsum of the hand, encompassing the area in which the stimulation of $A \beta$ afferents elicited the paresthesia. The laser beam was transmitted through an optic fiber, and its diameter at target site was set at $\sim 6 \mathrm{~mm}$ by focusing lenses. A visible $\mathrm{He}-\mathrm{Ne}$ laser pointed to the stimulated area, within which the laser beam was manually displaced after each stimulus. The laser was triggered by a computer script.

The method of ascending staircases used for identifying the detection threshold of $A \beta$ stimuli was also used to identify the detection threshold of $A \delta$ stimuli. For the EEG recordings, the stimulus energy was clearly above the activation threshold of $A \delta$ fibers $\left(0.53 \pm 0.06 \mathrm{~J} / \mathrm{mm}^{2}\right)$. This stimulus energy elicited intense but tolerable pinprick pain sensations, of comparable intensities on the right and left hands. Because variations in baseline skin temperature may modulate the intensity of the afferent nociceptive input (lannetti et al., 2004), an infrared thermometer was used to ensure that the hand temperature varied no $>1^{\circ} \mathrm{C}$ across blocks. To avoid receptor fatigue or sensitization, the laser beam was displaced after each stimulus by $\sim 1$ $\mathrm{cm}$ within the predefined stimulated area.

\section{Experimental procedure}

Participants sat comfortably with their hands resting on a table in front of them. They were instructed to focus their attention on the stimuli and fixate a yellow circular target (diameter: $1 \mathrm{~cm}$ ) placed in front of them at a distance of $\sim 60 \mathrm{~cm}$ from their face. A black curtain blocked the view of the hands. Throughout the experiment, white noise was played through headphones, to mask any sound associated with the either type of somatosensory stimulation.

The experiment was performed on 32 participants, divided in two groups of 16 participants. One group received electrical stimuli, and the other group received laser stimuli, using an identical procedure. Each participant received the somatosensory stimuli in 10 blocks, separated by a 5 -min interval, during which participants were allowed to rest. Each block consisted of 60 somatosensory stimuli delivered at $1 \mathrm{~Hz}$ : thus, each block lasted $1 \mathrm{~min}$. In each block, stimuli were delivered either to the right hand or to the left hand. Right- and left-hand blocks were alternated. The order of blocks was balanced across participants; half of the subjects started with a right-hand block, and the other half started with a left-hand block. At the end of each block, participants were asked to provide an average rating of perceived stimulus intensity, with reference to the modality of the stimulus and using a numerical scale ranging from 0 ("no shock sensation" or "no pinprick sensation") to 10 ("most intense shock sensation" or "most intense pinprick sensation"). This was done to ensure that the perceived intensity of the stimuli was similar across blocks (rating variability, SD across blocks: electrical stimuli, $0.2 \pm 0.2$; laser stimuli: $0.3 \pm$ $0.4)$.

\section{Electrophysiological recordings}

EEG was recorded using $30 \mathrm{Ag}-\mathrm{AgCl}$ electrodes placed on the scalp according to the International 10-20 system (Electro-Cap International), using the nose as reference. Electrode positions were Fp1, Fpz, Fp2, F7, F3, Fz, F4, F8, T3, C3, Cz, C4, T4, T5, P3, Pz, P4, T6, O1, Oz, O2, FCz, FC4, FC3, Cp3, Cp4. Eye movements and blinks were recorded from the right orbicularis oculi muscle, using two surface electrodes. The active electrode was placed below the lower eyelid, and the reference electrode a few centimeters laterally to the outer canthus. Signals were amplified and digitized using a sampling rate of $1024 \mathrm{~Hz}$ (SD32; Micromed).

\section{EEG analysis}

\section{Preprocessing}

EEG data were preprocessed and analyzed using MATLAB R2016b, Letswave 6 and EEGLAB (https://sccn.ucsd.edu/eeglab/). Continuous EEG data were bandpass filtered from 0.5 to $30 \mathrm{~Hz}$ using a Butterworth filter, segmented into epochs using a time window ranging from -0.2 to $0.8 s$ relative to the onset of each stimulus, and baseline corrected using the interval from -0.2 to $0 \mathrm{~s}$ as reference. Trials contaminated by large artefacts $(<10 \%$ per condition) were removed. Eye blinks and movements were corrected using a validated method based on unconstrained independent component analysis ("runica" algorithm of EEGLAB). In all datasets, independent components related to eye movements showed a large EOG channel contribution and a frontal scalp distribution. To allow averaging across blocks while preserving the possibility of detecting lateralized EEG activity, scalp electrodes were flipped along the medio-lateral axis for all signals recorded in response to left hand stimulation. Hereinafter, we refer to the central electrode contralateral to the stimulated hand as Cc. In each participant, we averaged each of the 60 ERP responses across the 10 recording blocks, and thus obtained 60 average ERP waveforms: one for each of the 60 trials and for each participant.

\section{Statistical assessment of ERP components}

We assessed the consistency of stimulus-evoked modulations of EEG amplitude across time, to statistically evaluate whether EEG deflections in the post-stimulus time window (from 0 to $+0.8 \mathrm{~s}$ ) were significantly greater than baseline. Specifically, we performed a one-sample, 
A $\beta$-ERPs

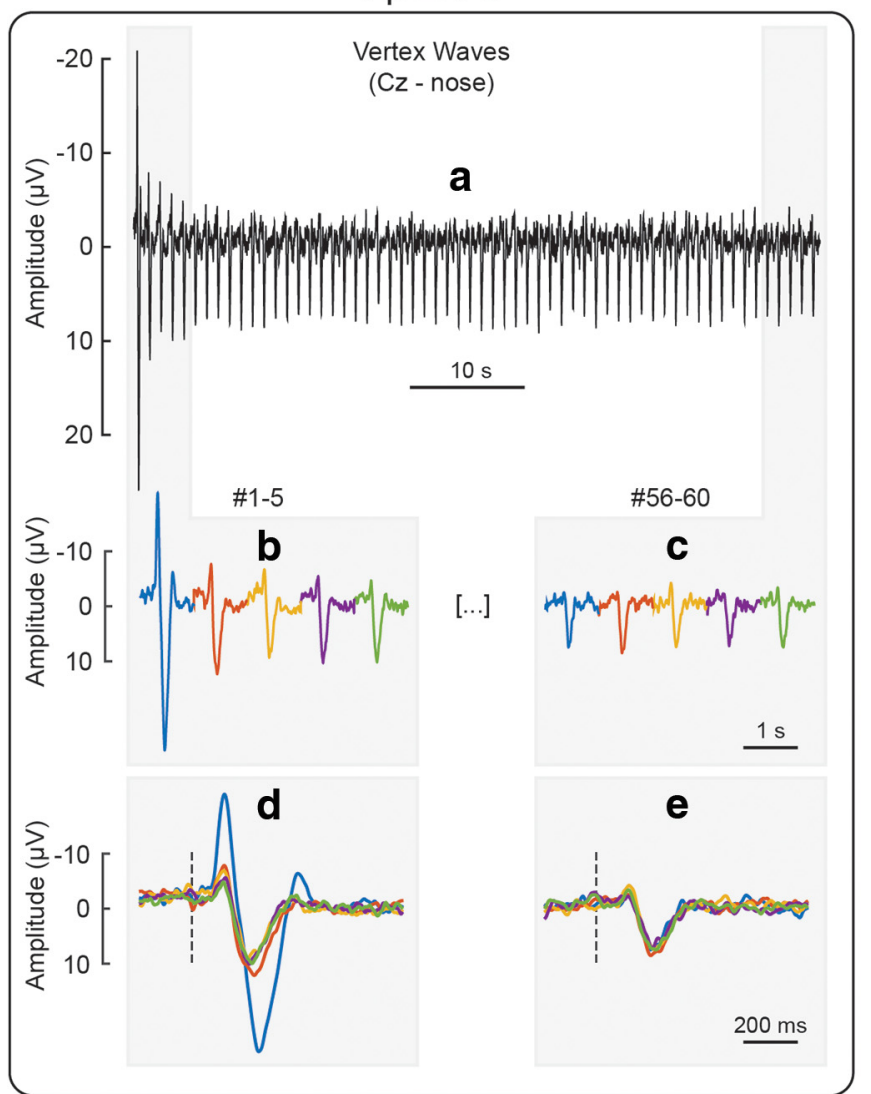

Ad-ERPs

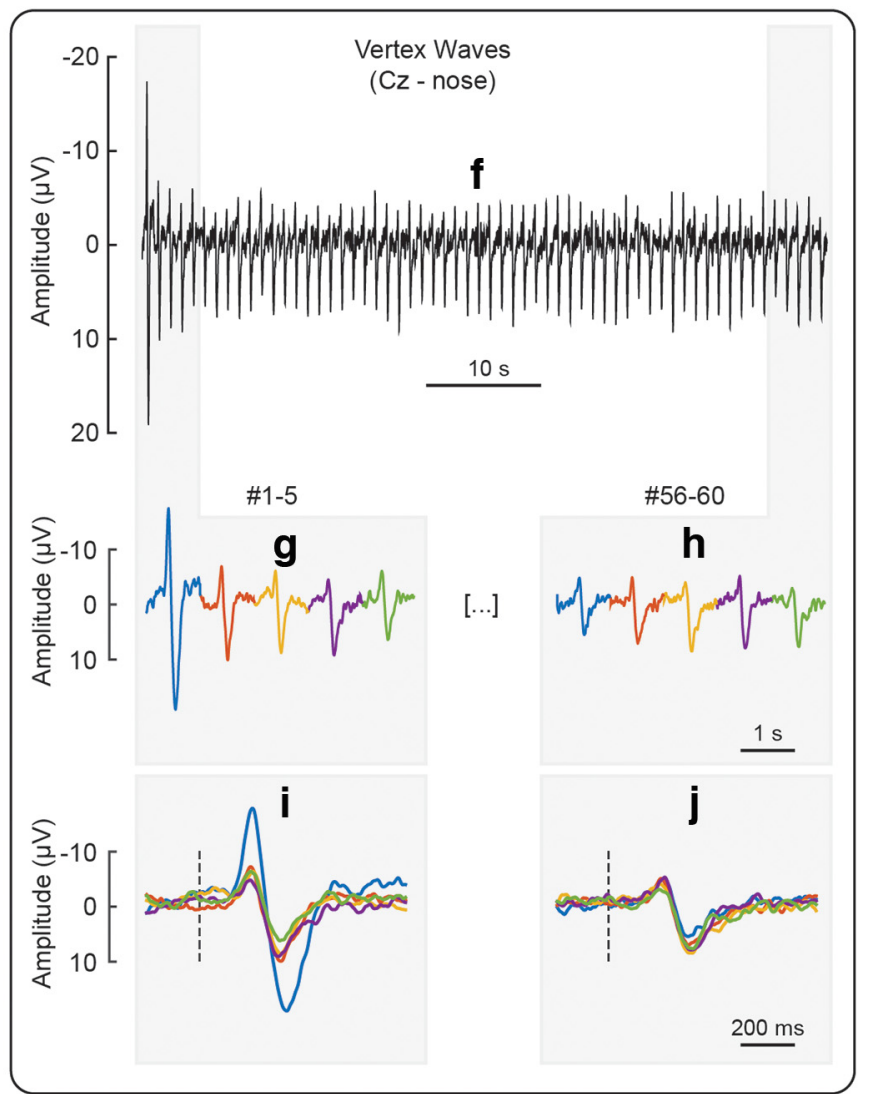

Figure 1. Habituation of VWs (N2, P2) elicited by repeated $A \beta$ (panels a-e) and A $\delta$ stimuli (panels $\boldsymbol{f}-\boldsymbol{j}$ ), at electrode $\mathrm{Cz}$ referenced to the nose. Panel a shows the VWs elicited by $60 \mathrm{~A} \beta$ stimuli delivered at $1 \mathrm{~Hz}$, whereas panel $\boldsymbol{f}$ shows the VWs elicited by $60 \mathrm{~A} \delta$ stimuli delivered at the same frequency. To facilitate visual comparison, the figure displays, enlarged and concatenated, the responses to the first five $A \beta$ stimuli (panel $\boldsymbol{b}$ ), the last five $A \beta$ stimuli (panel $\boldsymbol{c}$ ), the first five A $\delta$ stimuli (panel $\boldsymbol{g}$ ), and the last five $A \delta$ stimuli (panel $\boldsymbol{h}$ ). The figure also displays, enlarged and superimposed, the same responses to the first five $A \beta$ stimuli (panel $\boldsymbol{d}$ ), the last five $A \beta$ stimuli (panel $\boldsymbol{e}$ ), the first five A $\delta$ stimuli (panel $\boldsymbol{i}$ ), and the last five A $\delta$ stimuli (panel $\boldsymbol{j}$ ).

non-parametric, Wilcoxon signed-rank test against zero for each time point of the entire baseline-corrected, single-subject waveforms, using cluster-level permutation testing (Maris and Oostenveld, 2007; Van Den Broeke et al., 2015). A non-parametric test was chosen over a parametric test to not make the assumption that the data were normally distributed. The nonparametric clusterbased permutation approach for statistical testing assumes that true neural activity will tend to generate signal changes over contiguous time points. First, the EEG waveforms of the different conditions were compared by means of a point-by-point, one-sample Wilcoxon signedrank test against zero. Then, clusters of contiguous time points above the critical $z$ score for a non-parametric two-sided test were identified, and an estimate of the magnitude of each cluster was obtained by computing the sum of the $z$ scores constituting each cluster (cluster-level statistic). Random permutation testing (1000 times) of the subject-specific ERP waveforms of the different conditions (performed independently for every subject) was then used to obtain a reference distribution of mean cluster magnitude. Finally, the proportion of random partitions that resulted in a larger cluster-level statistic than the observed one was calculated. Clusters in the ob- served data were regarded as significant if they had a magnitude exceeding the threshold of the 2.5th and 97.5th percentile (corresponding to a two-sided test). This analysis was performed separately on the non-habituated ERP and on the habituated ERP of each modality.

The non-habituated ERP was derived, for each participant, by averaging the responses elicited by the 1st stimulus of all blocks. The habituated ERP was derived, for each participant, by averaging the responses elicited by the 6th to the 60th stimuli of all blocks. The decision of using these responses elicited by stimuli 6th to 60th as a proxy of the habituated ERP was based on the observation that the amplitude of the main ERP waves decays only minimally after the first five stimulus repetitions, as observed here (Figs. 1, 2, 4) and previously described (Fruhstorfer et al., 1970; Greffrath et al., 2007). Figures 1, 2 show how the amplitude of the ERPs was consistently habituated after the first few stimulus repetitions.

\section{Modeling the within-block decay of the lateralized and VWs}

We tested whether and how the amplitude of the VWs and of the lateralized wave evoked by $A \beta$ and $A \delta$ stimuli was modulated as a function of stimulus repetition. In 
A $\beta$-ERPs

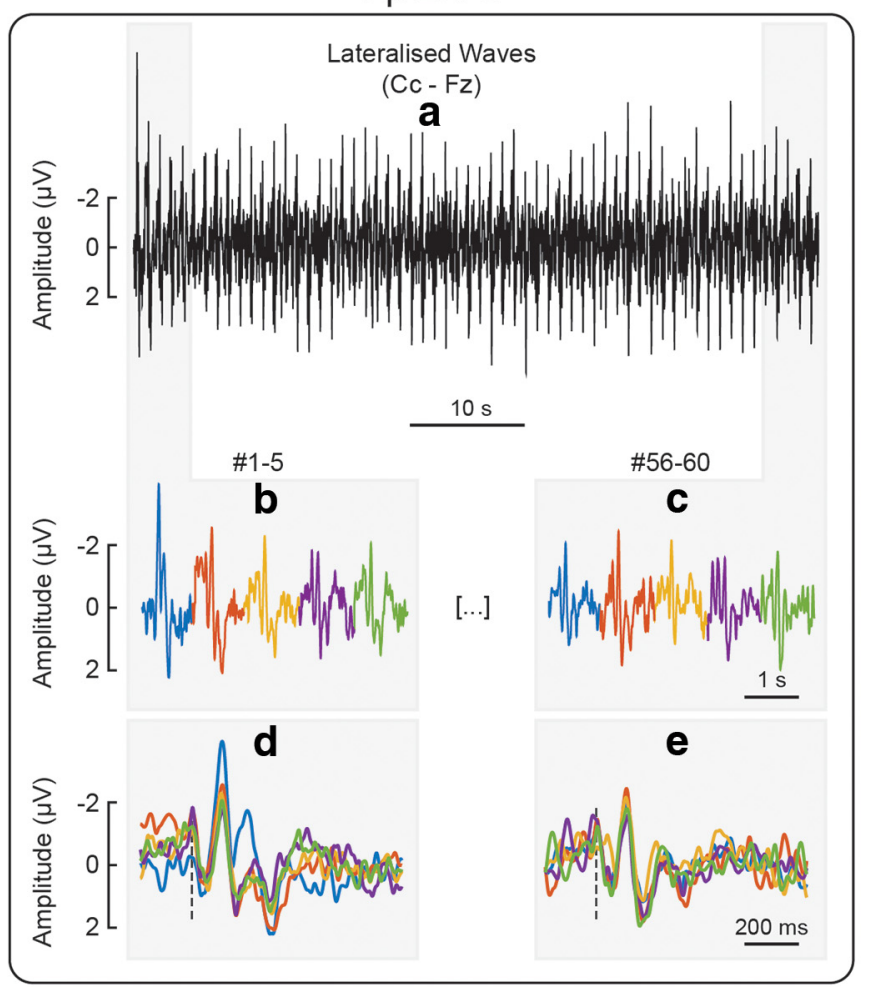

A $\delta$-ERPs

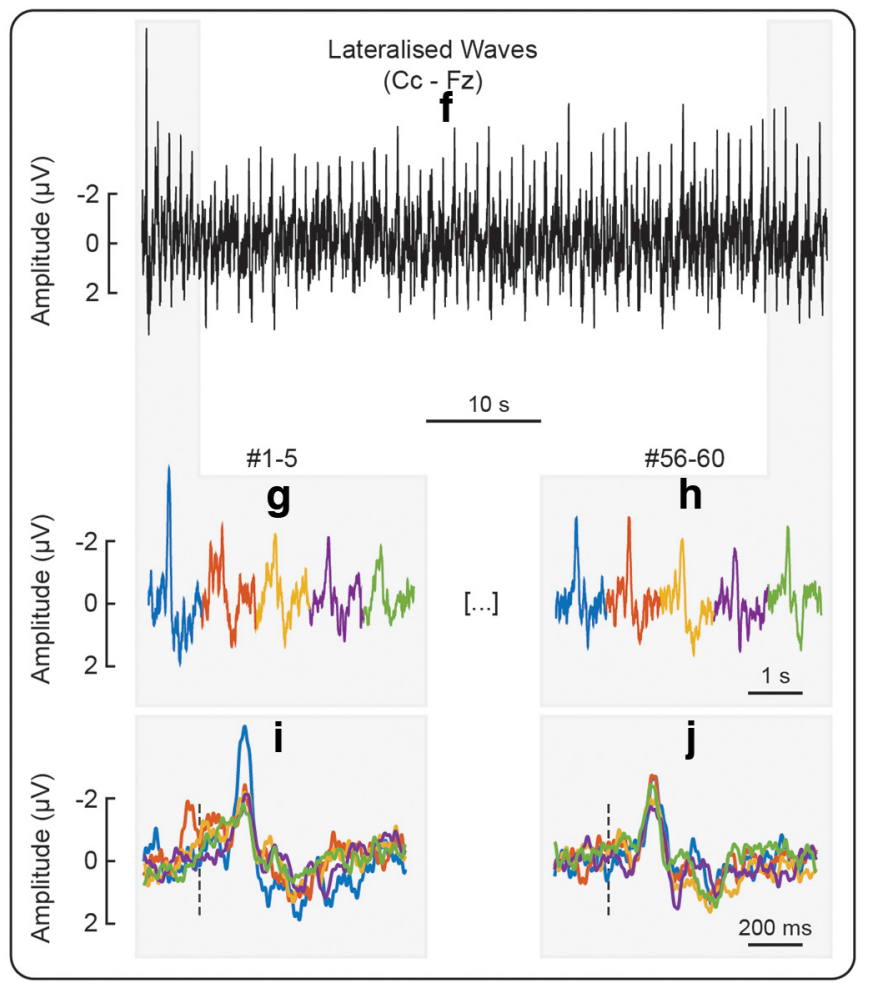

Figure 2. Habituation of the lateralized somatosensory waves $(N 1, P 4)$ elicited by repeated $A \beta$ (panels a-e) and A $\delta$ stimuli (panels $\boldsymbol{f}-\boldsymbol{j})$, at the central electrode contralateral to hand stimulation (Cc) referenced to Fz. Panel a shows the lateralized waves elicited by $60 \mathrm{~A} \beta$ stimuli delivered at $1 \mathrm{~Hz}$, whereas panel $\boldsymbol{f}$ shows the lateralized waves elicited by $60 \mathrm{~A} \delta$ stimuli delivered at the same frequency. To facilitate visual comparison, the figure displays, as enlarged and concatenated, the responses to the first five $A \beta$ stimuli (panel $\boldsymbol{b}$ ), the last five $A \beta$ stimuli (panel $\boldsymbol{c}$ ), the first five $A \delta$ stimuli (panel $\boldsymbol{g}$ ), and the last five $A \delta$ stimuli (panel $\boldsymbol{h}$ ). The figure also displays, as enlarged and superimposed, the same responses to the first five $A \beta$ stimuli (panel $\boldsymbol{d}$ ), the last five $A \beta$ stimuli (panel $\boldsymbol{e}$ ), the first five $A \delta$ stimuli (panel $\boldsymbol{i}$ ), and the last five A $\delta$ stimuli (panel $\boldsymbol{j}$ ).

each participant, we first averaged each of the $60 \mathrm{ERP}$ responses across the 10 recording blocks, and thus obtained 60 average ERP waveforms: one for each of the 60 trials. Then, we averaged across participants and, for each modality, we obtained 60 group-level averages. To study the amplitude modulation of the entire wave form across 60 trials, we decomposed the EEG signals at electrodes of interest ( $\mathrm{Cz}$ and $\mathrm{Cc}$ ) using SVD (Golub and Reinsch, 1970). We used SVD to decompose the modulation of the EEG amplitude across the 1000-ms epoch (which give rise to the ERP wave) from the modulation of the EEG amplitude across 60 trials.

SVD is a method for decomposing the data matrix $\mathbf{M}(s \times e)$, in this case EEG signals: $s=1024$ time samples, $e=60$ trials (given that the sampling rate is $1024 \mathrm{~Hz}$, each 1000-ms epoch has 1024 samples) into $s$ wave components [left singular vectors, defined as the columns of a matrix $\mathbf{U}(s x s)$ ] and $e$ habituation components [right singular vectors, defined as the columns of a matrix $\mathbf{V}(e x e)$ ]. The left-singular vectors tell us how the EEG amplitude is modulated across the 1000-ms epoch (wave component), and the right-singular vectors describe how the EEG amplitude is modulated across 60 trials (habituation component). Each left-right component pair is multiplied by a scaling factor $\sigma$, and pairs are rank-ordered according to those factors, where the most important pairs correspond to the largest values of $\sigma$, and the least important ones (typically noise) correspond to the lowest $\sigma$. Formally, SVD is given by $\mathbf{M}=\mathbf{U} \sum \mathbf{V}^{\boldsymbol{\top}}$, where $\sum$ is a $s x$ e diagonal matrix with the scaling factors on the diagonal (singular values), $\mathbf{U}$ and $\mathbf{V}$ are the matrices of left and right singular vectors, respectively, and $\mathbf{V}^{\mathbf{T}}$ is the matrix transpose of $\mathbf{V}$. The first component pair gives the optimal rank-1 approximation to the original data matrix, in the least square sense. The first two components give the optimal rank-2 approximation, and so on and so forth.

To test the significance of the SVD decomposition, we separated the variance caused by stimulus-evoked activity from other types of variance (noise), and performed the SVD on the noise traces; finally, we tested whether the results of the SVD performed on the noise traces were different from the SVD performed on $\mathbf{M}$ (which contains a mixture of signal and noise), adapting an approach previously described (Sengupta and Mitra, 1999; Machens et al., 2010).

Specifically, for each subject and condition, we first estimated the residual noise traces $\eta_{\mathrm{i}}(s, e)$, by taking the average of the differences between the single-subject EEG amplitude $y_{i}(s, e)$ and group-average EEG amplitude $\mathrm{Y}_{-\mathrm{i}}(s, e)$ (the group average was calculated after excluding subject i): 


$$
\eta_{\mathrm{i}}(s, e)=\mathrm{y}_{\mathrm{i}}(\mathrm{s}, e)-\mathrm{Y}_{-\mathrm{i}}(\mathrm{s}, e)
$$

We then performed SVD on the residual noise traces $\eta$ $(s, e)$, for each subject and condition. We averaged the resulting $\mathbf{U}_{\text {noise, }}, \sum_{\text {noise }}, \mathbf{V}^{\boldsymbol{\top}}$ noise across subjects and divided them by the square root of the number of subjects. We also calculated their SEM. We tested the significance of the ranks of $\sum$ by comparing whether each diagonal value of $\sum$ was greater than the corresponding value of $\left[\sum_{\text {noise }}+2.33 \mathrm{SE}\right]$ : this corresponds to a one-tail test at a $p=0.01$. Lastly, we tested the significance of $\mathbf{U}$ and $\mathbf{V}^{\mathbf{T}}$ by comparing whether their value at each rank was different (either greater or lower) than the corresponding value of $\left[\mathbf{U}_{\text {noise }} \pm 2.58 \mathrm{SEM}\right]$ and $\left[\mathbf{V}^{\boldsymbol{\top}}{ }_{\text {noise }} \pm 2.58 \mathrm{SEM}\right]$ : this corresponds to a two-tails test at a $p=0.01$.

Finally, we modeled the amplitude modulation across trials (habituation components) by fitting the following models to the right-singular vectors at each eigenvalue scale factor (or rank order):

(1) $y=a+b / x$

(2) $y=a+b / x^{c}$

(3) $y=a+b e^{-c x}$

(4) $y=c$

where $y$ is the peak amplitude of each given ERP wave, $x$ is the trial number (from 1 to 60), $e$ is the Euler constant, and $a, b, c$ are the parameters to be estimated using a non-linear least squares method. We tested these specific models of ERP decay (\#1-\#3) given the previous evidence that the VW decays sharply at the first stimulus repetition (Fruhstorfer et al., 1970; Greffrath et al., 2007; lannetti et al., 2008; Valentini et al., 2011; Ronga et al., 2013). Note that model (4) corresponds to the absence of habituation, and fitting this model simply gives $\mathrm{c}$ equal to the mean of y. To compare which model best fitted the data, we calculated the Bayesian Information Criterion (BIC) of each model for each component, ordered by rank. The BIC allows a fair comparison between models of different complexity because it penalizes models with more parameters (Cover and Thomas, 2006). The lower the BIC, the better the model represents the measured data. For each component rank, we calculated the probability of rejecting the null hypothesis that there was no habituation (i.e., model \#4 best represents the data) and accepting the alternative hypothesis that there was significant habituation (i.e., either model \#1, \#2, or \#3 wins), by using a resampling approach with 1000 iterations: at each iteration, we shuffled the order of epochs, fitted models \#1-\#4, and compared the goodness of fit according to BIC.

\section{Code accessibility}

The code described above in Statistical assessment of ERP components and Modeling the within-block decay of the lateralized and VWs was written in MATLAB 2016b and is freely available online at https://github.com/flamancini/ERP_habituation_2018. The code is also available as Extended Data.

\section{Data availability}

Pre-processed EEG data are publicly available at https://osf.io/8wj3s/.

\section{Results}

\section{Response waveforms and topographies}

Group-average ERPs elicited by $\mathrm{A} \beta$ and $\mathrm{A} \delta$ stimuli are shown in Figures 1-3. As expected, the latency of $A \delta$ ERPs was longer than the latency of $A \beta$-ERPs, because A $\delta$ fibers are thinly myelinated and thus have slower conduction velocity than large-myelinated $A \beta$ fibers (Mountcastle, 2005).

Figure 1 shows that the amplitude decay of the negative and positive VWs (N2 and P2, Cz vs nose) elicited by the 60 repeated somatosensory stimuli, whereas Figure 2 shows the amplitude modulation of the lateralized somatosensory waves (N1 and P4, Cc vs Fz). To facilitate visual inspection, we enlarged the responses to the first five and last five stimuli (same responses presented both concatenated and superimposed in Figs. 1, 2). Figure 3 demonstrates that, both in the non-habituated response (trial \#1, Fig. 3a,c) and in the habituated response (average of trials \#6-\#60, Fig. $3 b, d)$, the N2 and P2 waves were greater than baseline. Not only they survived 1-min of repeated stimulation, but clearly dominated the majority of the ERP responses.

In both stimulus modalities, the lateralized somatosensory waves were much smaller than the VWs, as expected (Valentini et al., 2012; Hu et al., 2014), and the identification of the P4 peak was ambiguous for the A $\beta$-ERP elicited by trials $6-60$ (Fig. $3 A$ ). Importantly, albeit small in amplitude, both the early $\mathrm{N} 1$ and the late P4 lateralized waves elicited in trials 1 and 6-60 were nevertheless consistently greater than baseline, as demonstrated by the point-by-point non-parametric tests reported in Figure 3. The peaks of the $\mathrm{N} 1$ waves elicited in trials 1 (Fig. $3 a, c$ ) and 6-60 (Fig. 3b,d) had maximal spatial distribution over the central electrodes in the hemisphere contralateral to hand stimulation (Fig. 3), as shown in previous studies ( $\mathrm{Hu}$ et al., 2014; Mancini et al., 2015).

\section{Modeling the within-block decay of the lateralized waves and VWs}

We took a modeling approach to decompose the modulation of the EEG amplitude across the 1000-ms epoch (which give rise to the ERP wave) from the modulation of the EEG amplitude across 60 trials. This analysis has the benefit of providing an optimal, rank-based approximation to the original data matrix, allowing us to detect habituation effects. Figures 4, 5 display the results of the SVD analyses performed at channels $\mathrm{Cz}$ (VWs) and Cc (lateralized waves) respectively, elicited by non-nociceptive A $\beta$ stimuli (Figs. $4 a, 5 a$ ) and nociceptive A $\delta$ stimuli (Figs. $4 b, 5 b$ ). The singular values can be considered as the scaling factors of the left-singular and right-singular vectors. The left-singular vector shows whether and how the EEG amplitude was modulated within the 1000-ms epoch, and right-singular vector shows whether and how the EEG amplitude was modulated across 60 trials. The noise distribution for singular, left-singular, and rightsingular vectors is shown in red (with 99\% confidence intervals). Figure 6 summarizes which model best fitted the EEG amplitude modulation across trials, at each rank and according to BIC. 
Trial \#1
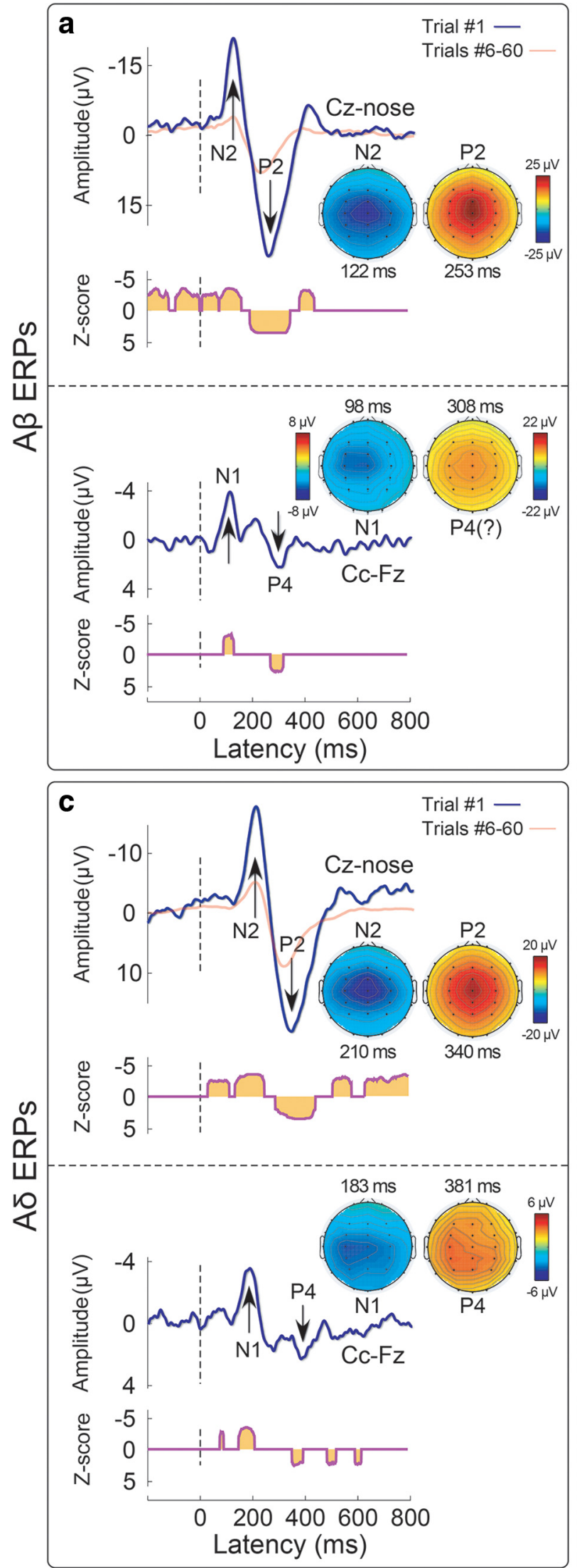

Trials \#6-60
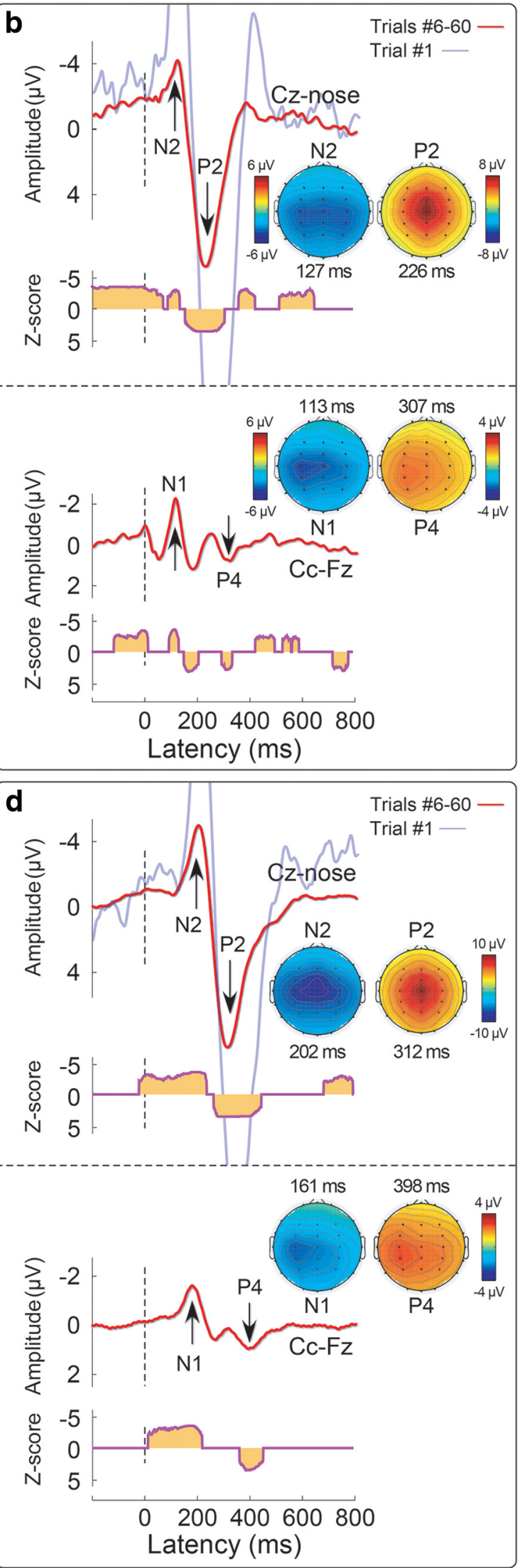

Figure 3. Habituation of VWs (N2, P2) and lateralized responses (N1, P4) elicited by A $\beta$ (panels $\boldsymbol{a}, \boldsymbol{b}$ ) and A $\delta$ (panels $\boldsymbol{c}, \boldsymbol{d}$ ) 
continued

somatosensory stimuli. Displayed signals show group-level ERPs recorded from the vertex (Cz vs nose) and from the central electrode contralateral to the stimulated hand (Cc vs Fz), elicited by the first stimulus in a series (non-habituated response; panels $a, c$ ) and by the average of trials \#6-\#60 (habituated response; panels $\boldsymbol{b}$, $\boldsymbol{d}$ ). Scalp topographies (signals referenced to the nose) are displayed at the peak latency of the N1, N2, P2, and P4 waves, in all conditions. The N1, N2, and P2 waves were significantly $>0$ both in trial \#1 and in trials \#6-\#60, as shown by the point-by-point, cluster-corrected $(p<0.05$, two tails), one-sample Wilcoxon z-statistics plotted below each ERP wave.

The amplitude modulation of the VWs elicited by $A \beta$ stimuli was significantly described by the first two ranks (Fig. 4A): the first two singular values were greater than the singular values of the noise distribution (at $p=0.01$ ). The modulation of the EEG amplitude within the epoch (left-singular vectors) had the characteristic shape of the VW at the first two ranks (Fig. 4A). The latency of the peaks of these waveforms fell clearly within the range of the N2 and P2 peak latencies (Fig. 4A, left-singular vector; compare Fig. $3 a, b)$ : the peaks of the left-singular vector at the first rank had a latency of $125 \mathrm{~ms}$ (corresponding to the $\mathrm{A} \beta$-N2 peak) and $225 \mathrm{~ms}$ (corresponding to the $\mathrm{A} \beta$-P2 peak); the peaks at the second rank had a latency of $196 \mathrm{~ms}$ (corresponding to the late part of the $A \beta-\mathrm{N} 2$ wave) and $292 \mathrm{~ms}$ (corresponding to the late part of the $\mathrm{A} \beta-\mathrm{P} 2$ wave). Furthermore, the EEG amplitude elicited by $A \beta$ stimuli decayed significantly across trials at the first two ranks (Fig. $4 A$, right-singular vector). The winning decay models $(y=a+b / x)$ are displayed with a black line superimposed onto the right-singular vectors, and their $p$ values were $<0.001$ at rank- 1 and 0.012 at rank-2.

The signal decomposition of the VWs elicited by nociceptive A $\delta$ stimuli is reported in Figure $4 B$. Only the first rank of singular values was greater than noise: at the first rank, the modulation of the EEG amplitude had the characteristic shape and latency of the VW (Fig. 4B, leftsingular vector): the peaks of the left-singular vector at the first rank had a latency of $202 \mathrm{~ms}$ (corresponding to the peak of the $\mathrm{A} \delta$-N2) and $317 \mathrm{~ms}$ (corresponding to the peak of the $A \delta$-P2; compare Fig. $3 c, d)$. Although the EEG amplitude clearly decreased from the first to the second trial at the first rank, the fitting of decay models was not significant (Fig. 4B, right-singular vector). Although the second rank of singular values was not significantly different from noise, the modulation of EEG amplitude across time samples was greater than noise at a latency of $270 \mathrm{~ms}$ (corresponding to the late part of the N2 wave) and $380 \mathrm{~ms}$ (Fig. 4B, left-singular vector): the amplitude of the second-rank component was greater than noise only at the first trial, and its decay was best modeled by the same decay function that described the decay of the VW elicited by A $\beta$ stimuli $(y=a+b / x ; p=0.025)$.

The amplitude modulation of the lateralized somatosensory waves elicited by $A \beta$ stimuli (Fig. $5 A$ ) and $A \delta$ stimuli (Fig. 5B) was described by the first rank of singular values $(p<0.01)$. At the first rank, the peak of the left-singular vector fell within the range of the peak amplitude of the N1 wave, both for $A \beta$ stimuli (112 ms) and for $A \delta$ stimuli (181 ms; Fig. 5A,B; compare Fig. 3). At the second-rank, the left-singular vector for $A \beta$ stimuli was characterized by two peaks significantly greater than noise: the earliest peak latency fell within the range of the $A \beta-N 1$ peak latency $(112 \mathrm{~ms})$, whereas the second peak had a latency longer than the $\mathrm{A} \beta-\mathrm{N} 2$ and shorter than the $\mathrm{A} \beta-\mathrm{P} 2$ peaks (184 ms; compare Fig. 3a,b). The amplitude of the EEG responses elicited by $A \beta$ stimuli at the first rank was greater than noise (Fig. $5 A$, right-singular vector), but did not habituate across trials (i.e., the non-habituation model best fitted the right-singular vector). However, at the second rank, the EEG amplitude of the first three trials was greater than noise, and the signal habituation was again in the form of $y=a+b / x$ (Fig. $5 A$, right-singular vector; $p=$ 0.059 ). Finally, the ERP elicited by A $\delta$ stimuli significantly habituated across trials: indeed, the right-singular vector at the first rank habituated following the same decay functions of the $\mathrm{N} 2$ and $\mathrm{P} 2$ waves elicited by $\mathrm{A} \beta$ stimuli and $A \delta$ stimuli $(y=a+b / x ; p=0.027$; see also Fig. 6$)$.

\section{Discussion}

In this study, we characterized the habituation of the different components of the ERPs elicited by 60 identical somatosensory stimuli (activating either $A \beta$ non-nociceptive or $A \delta$ nociceptive primary afferents) delivered at 1 $\mathrm{Hz}$. Although the response amplitude was clearly reduced, the spatiotemporal sequence of the ERP waves was overall preserved in the habituated response (Fig. 3). This was substantiated by point-by-point statistical analysis: both lateralized somatosensory components and supramodal vertex components typically observed in the ERP elicited by sporadic and unpredictable stimuli (Liang et al., 2010; Hu et al., 2014; Mancini et al., 2015) also contributed to the ERP elicited by frequent and predictable stimuli. This result challenges a previous report that 60 repetitions of nociceptive stimuli at $1 \mathrm{~Hz}$ fully suppresses lateralized waves (Mouraux et al., 2013) and indicates that lateralized waves are obligatorily elicited by nociceptive-selective stimulation. Furthermore, we used SVD to decompose the modulation of the EEG amplitude across the 1000-ms epoch (which give rise to the ERP wave) from the modulation of the EEG amplitude across 60 trials. We found that the same model described the habituation of the VWs and lateralized waves elicited by $A \beta$ and $A \delta$ stimuli (Figs. 4-6): that was the simplest decay function in the form of $y=a+b / x$, where $y$ is the EEG amplitude, $x$ is the trial number, and $a, b$ are the estimated parameters. This indicates that the amplitude of both vertex and lateralized waves decays monotonically, with a largest, transient drop of response magnitude at the first stimulus repetition, followed by much smaller decreases in subsequent repetitions. 
A signal decomposition of vertex waves elicited by $\mathrm{A} \beta$ stimuli (channel $\mathrm{Cz}$ )

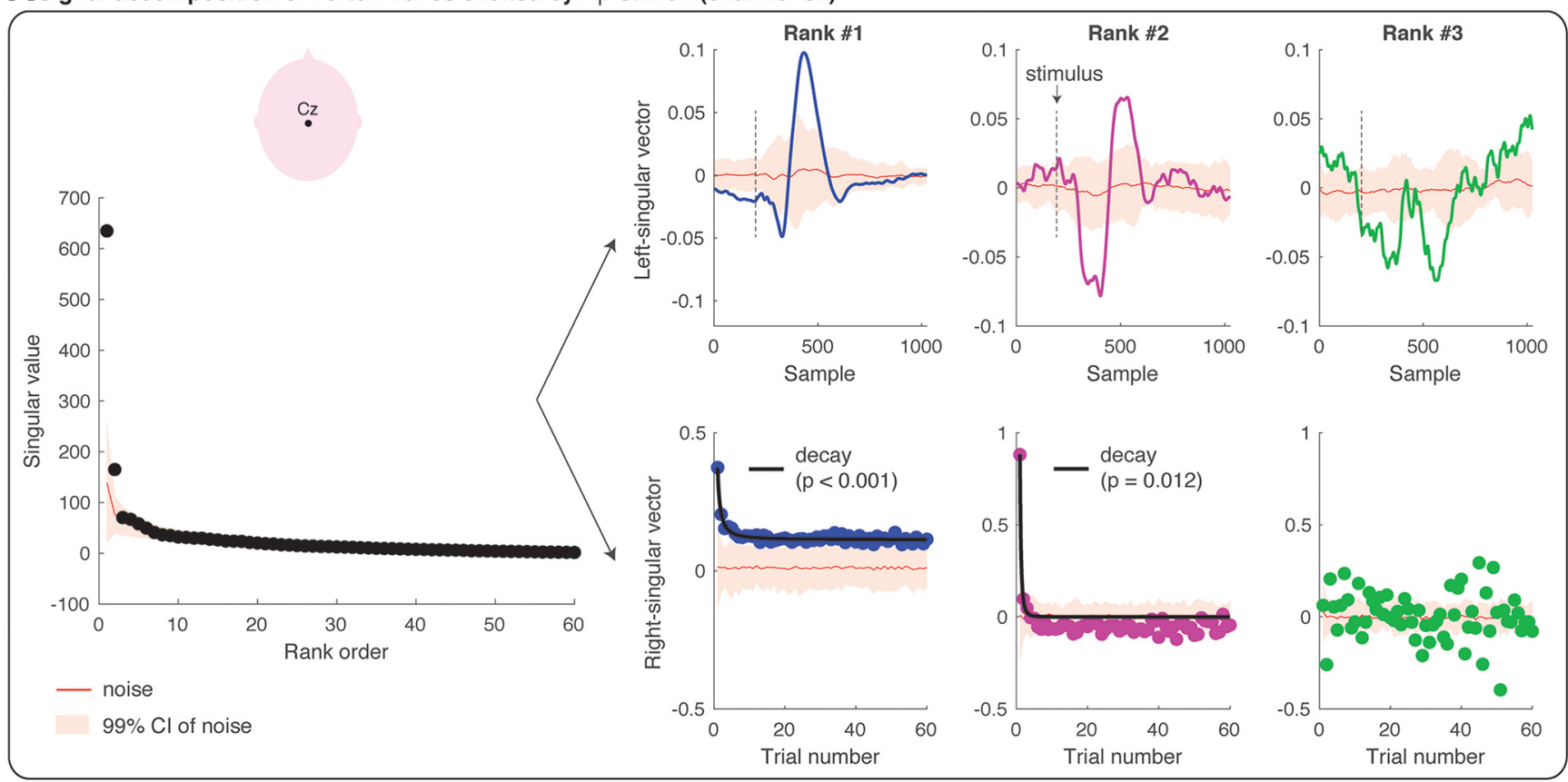

B Signal decomposition of vertex waves elicited by A $\delta$ stimuli (channel $\mathrm{Cz}$ )

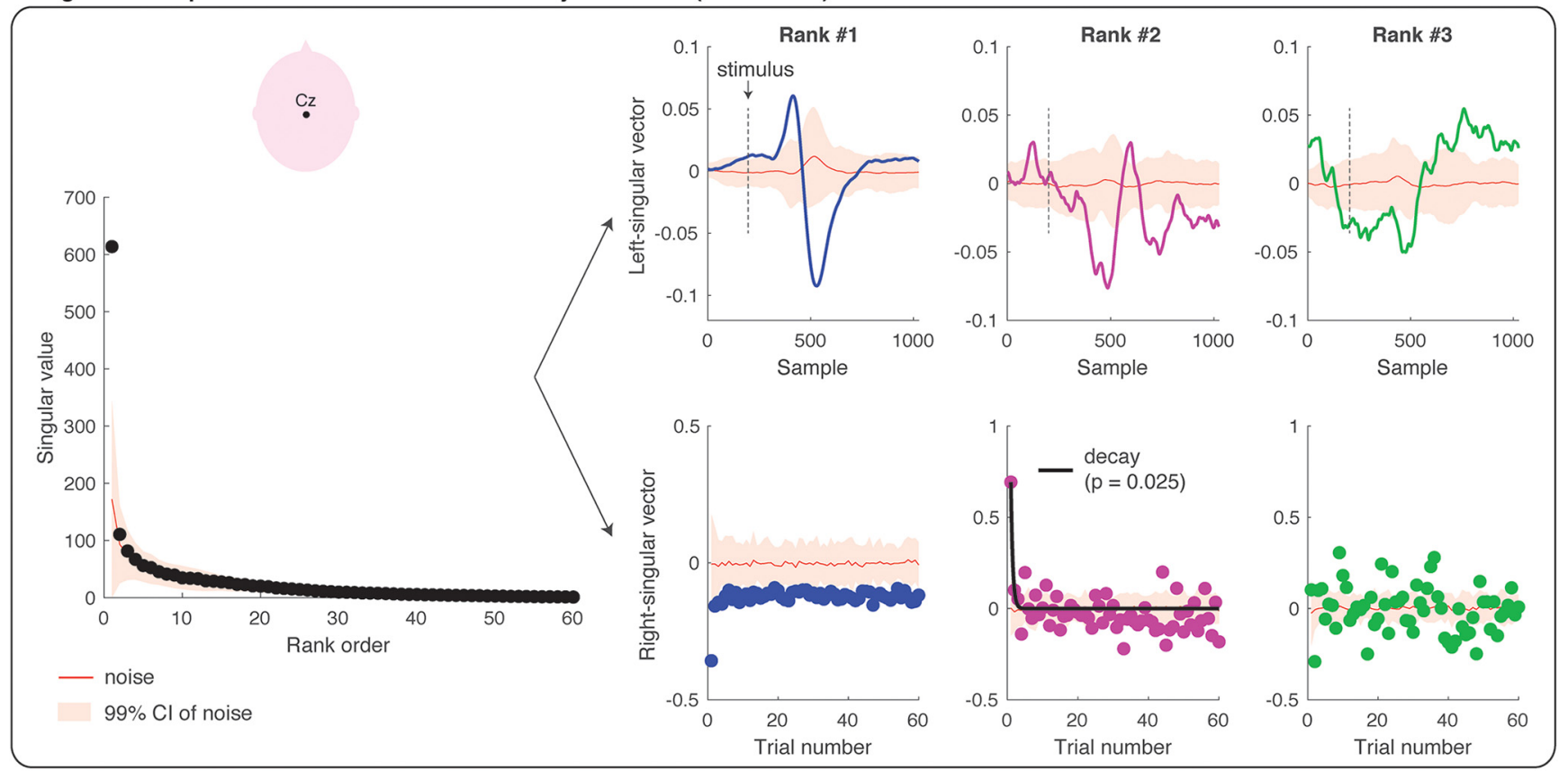

Figure 4. SVD and modeling of the amplitude modulation of the VWs (at channel Cz) elicited by repeated $A \beta$ (panel $\boldsymbol{A}$ ) and $A \delta$ (panel $B$ ) stimuli. Each figure panel displays the singular values at each of the 60 ranks, and the left- and right-singular vectors at the first three ranks. The singular values are the scaling factors of left- and right-singular vectors, and they are ranked according to their importance (from the most important to the least important). The left-singular vector shows the modulation of EEG amplitude across the epoch of $1000 \mathrm{~ms}$ (i.e., 1024 samples recorded at $1024 \mathrm{~Hz}$ ). The stimulus onset is marked with a dashed black line. The right-singular vector shows the modulation of EEG amplitude across the 60 trials. The red line in all plots shows the group-average results of the SVD of the single-subject residual noise traces, with a $99 \%$ confidence interval for statistical comparison $(p=0.01)$. Habituation models were fitted to the right-singular vectors at each rank. If a habituation model wins over a non-habituation model, the fit of the model is displayed with a black line superimposed on the right-singular vector values and the corresponding $p$ value is reported. In all the instances in which the non-habituation model wins over a habituation model, no fit is displayed. 
A Signal decomposition of lateralized waves elicited by A $\beta$ stimuli (channel Cc)

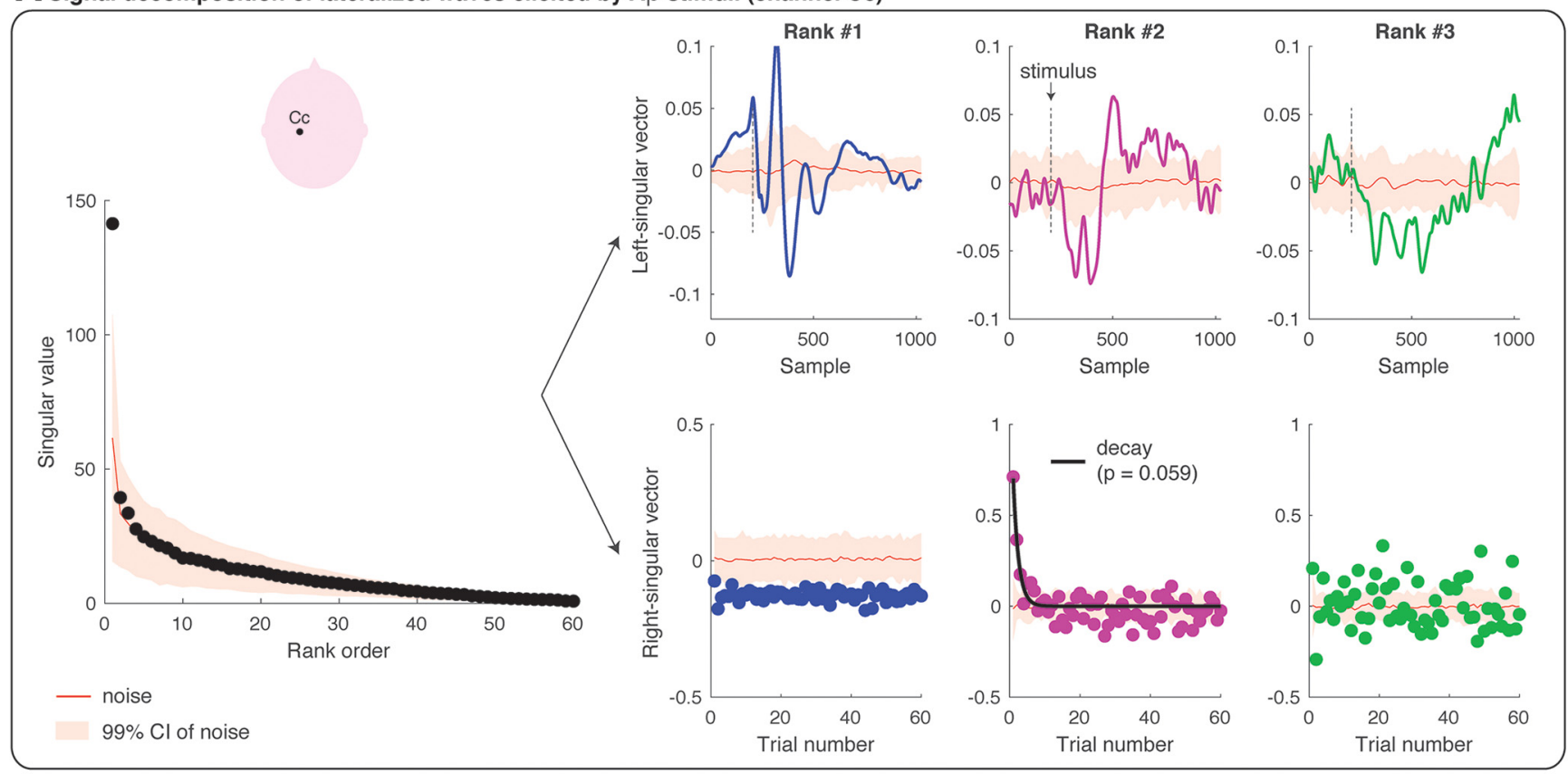

B Signal decomposition of lateralized waves elicited by A $\delta$ stimuli (channel Cc)

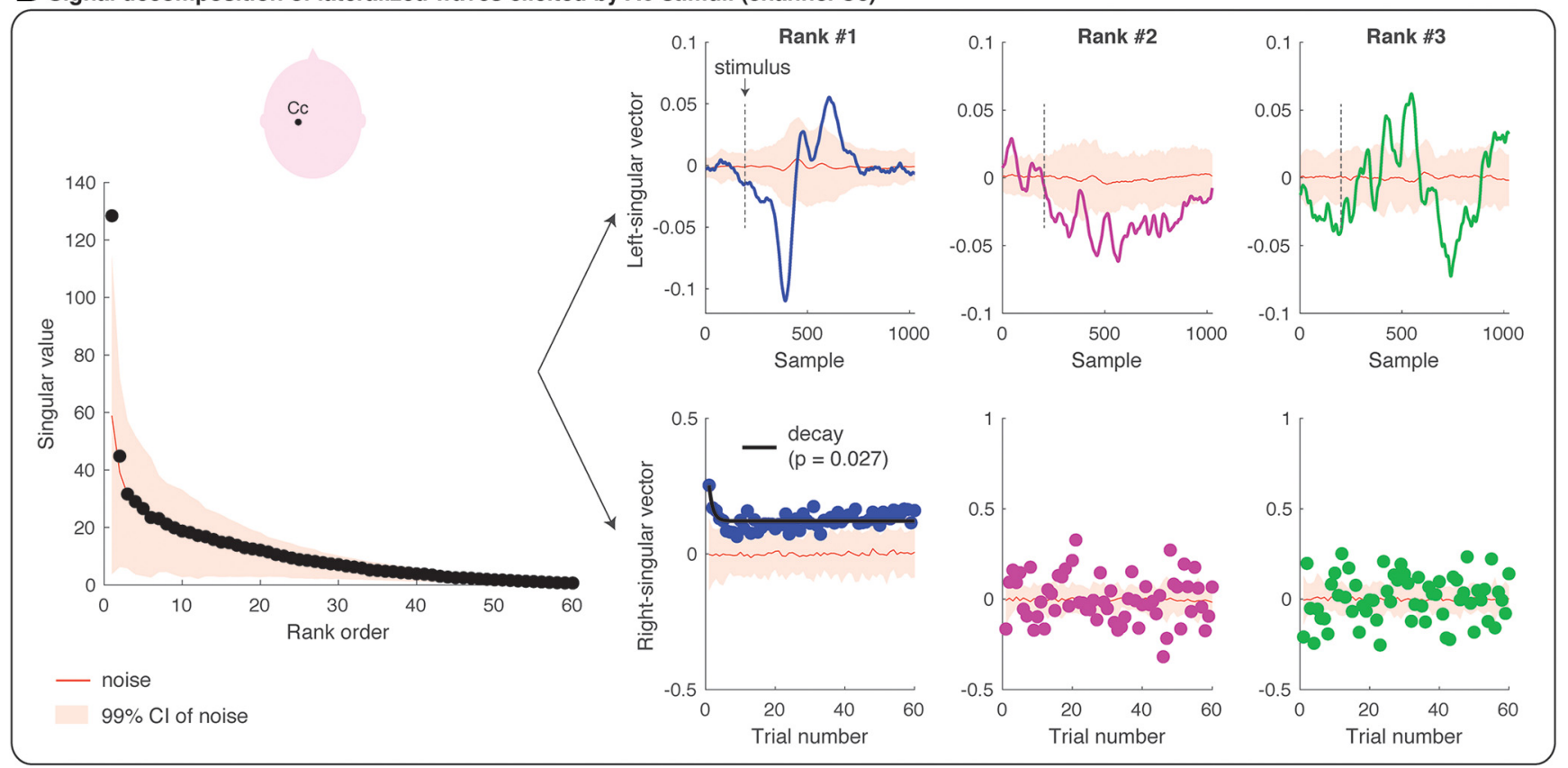

Figure 5. SVD and modeling of the amplitude modulation of the lateralized waves (at channel Cc) elicited by repeated A $\beta$ (panel $\boldsymbol{A}$ ) and $A \delta$ (panel $B$ ) stimuli. Each figure panel displays the singular values at each of the 60 ranks, and the left- and right-singular vectors at the first three ranks. The singular values are the scaling factors of left- and right-singular vectors, and they are ranked according to their importance (from the most important to the least important). The left-singular vector shows the modulation of EEG amplitude across the epoch of 1000 ms (i.e., 1024 samples recorded at $1024 \mathrm{~Hz}$ ). The stimulus onset is marked with a dashed black line. The right-singular vector shows the modulation of EEG amplitude across the 60 trials. The red line in all plots shows the group-average results of the SVD of the single-subject residual noise traces, with a $99 \%$ confidence interval for statistical comparison $(p=0.01)$. Habituation models were fitted to the right-singular vectors at each rank. If a habituation model wins over a non-habituation model, the fit of the model is displayed with a black line superimposed on the right-singular vector values and the corresponding $p$ value is reported. In all the instances in which the non-habituation model wins over a habituation model, no fit is displayed. 


\section{Winning models of habituation}

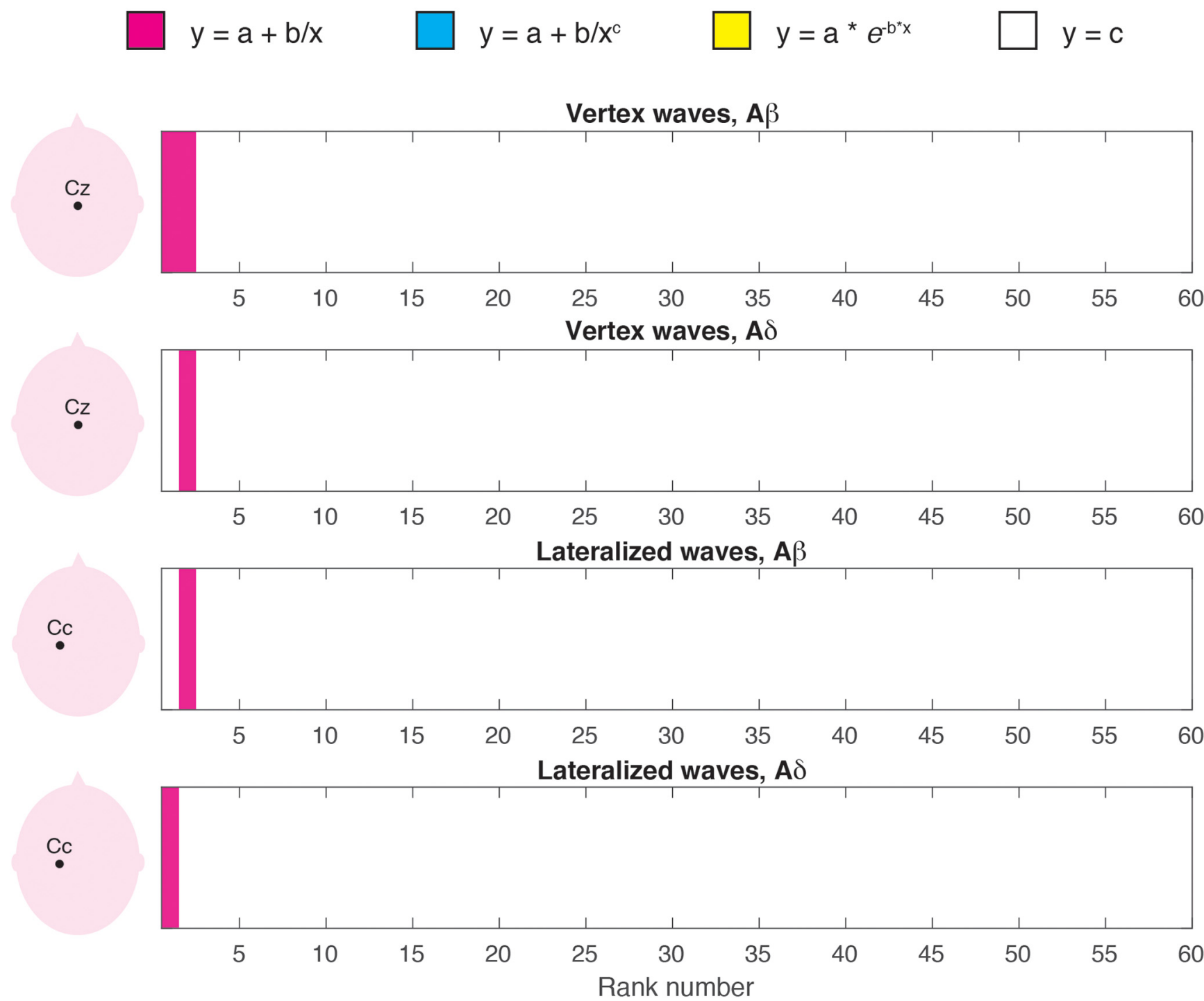

Figure 6. Winning model of ERP modulation by stimulus repetition. Following SVD, three habituation models and a non-habituation model were fitted to the right-singular vectors at each of the 60 ranks and compared according to BIC. The winning models are color-coded (pink: $y=a+b / x$; white: no habituation). Other decay models never win (blue, yellow).

\section{Effect of stimulus repetition on somatosensory lateralized responses}

In somatosensory ERPs, the VW is both preceded and followed by other deflections of smaller amplitude. These have a topographical distribution maximal over centroparietal electrodes in the hemisphere contralateral to hand stimulation. The earliest negative wave is usually referred to as N1 (Treede et al., 1988; Valentini et al., 2012) and the latest positive wave form of somatosensory ERPs is referred to as P4 (Hu et al., 2014; Mancini et al., 2015). Whereas the P4 has only been recently identified and its significance is not yet understood, the N1 has been described repeatedly in a large body of studies (Treede et al., 1988; Spiegel et al., 1996; Garcia-Larrea et al., 2003; Lee et al., 2009; Hu et al., 2014; Mancini et al.,
2015), and largely reflects somatosensory-specific neural activities (Lee et al., 2009; Liang et al., 2010).

The neural origin of the $\mathrm{N} 1$ wave has been long debated and remains unresolved, but it seems to be at least partially different in the ERPs elicited by non-nociceptive and nociceptive somatosensory stimuli (Garcia-Larrea et al., 2003; Ohara et al., 2004; Frot et al., 2013). A number of studies performing intracerebral recordings have indicated that the $\mathrm{A} \delta$ - $\mathrm{N} 1$ wave is largely contributed by the operculo-insular cortex (Frot et al., 1999; Peyron et al., 2002; Valeriani et al., 2004), whereas other studies have indicated that both the $\mathrm{N} 1$ and P4 waves can also be generated in the primary somatosensory cortex, both in human EEG and rodent ECoG recordings (Treede et al., 1988; Valentini et al., 2012; Hu et al., 2014; Jin et al., 
2018). For instance, a previous EEG study (Valentini et al., 2012) has demonstrated that the N1 elicited by nociceptive stimulation of the right and left hand have maximum scalp distribution over the central-parietal electrodes contralateral to the stimulated side. In contrast, the N1 elicited by nociceptive stimulation of the right and left foot are symmetrically distributed over the central-parietal midline electrodes (see also Treede et al., 1988; Jin et al., 2018). These findings are compatible with the somatotopic representation of the body in the primary somatosensory and motor cortex.

A novel result of our study is that these somatosensory $\mathrm{N} 1$ and $\mathrm{P} 4$ responses are detectable not only in the response to the first stimulus, but also in the habituated ERP response, as supported by the statistical assessment of the scalp distribution of the ERP response elicited by both the first and the last stimuli of the series (Fig. 3). This is important, given that a previous study using trains of intraepidermal electrical shocks at $1 \mathrm{~Hz}$ failed to observe any lateralized response (Mouraux et al., 2013). We note, however, that in this previous study nociceptive afferents were activated using intraepidermal electrical stimulation, which can cause strong peripheral and perceptual habituation, more significant than for radiant heat stimulation (Mouraux et al., 2010). Thus, in Mouraux et al. (2013) peripheral habituation induced by repeated intraepidermal electrical stimulation in the same skin location may have further reduced the already low signal-to-noise ratio of $\mathrm{N} 1$ and $\mathrm{P} 4$ waves.

Another novel result of our study is that the lateralized waves habituate across the 60 trials following the same decay functions of the VWs (Figs. 4-6). We used SVD not only to decompose the modulation of EEG amplitude within the block and across trials, but also to model the decay of an optimized model of EEG modulation. Indeed, SVD allows separating signals from noise (similarly to principal component analysis) and provides an optimized description of the ERP waves at the most informative ranks. This signal optimization allows characterizing the amplitude modulation of small and noisy ERP components.

A previous MEG study has reported that neural activity originating from primary somatosensory cortex is more resilient to stimulus repetition $(2-\mathrm{Hz}$ pneumatic stimulation of the fingers and face): in other words, it decays to a less extent and more slowly than neural activity in higher-order cortical regions, such as the posterior parietal cortex (Venkatesan et al., 2014). We used slower stimulus frequencies than these studies, so we cannot exclude that different time-scales of habituation may emerge at faster stimulus repetitions.

Finally, our design was not suited to investigate the habituation of the earliest sensory components of $A \beta$ ERPs (e.g. the N20 wave), which typically require averaging responses elicited by hundreds of stimuli. However, we note that the $\mathrm{N} 20$ wave of $A \beta$-ERPs, which originates in area $3 b$, is very resilient to stimulus repetition (García Larrea et al., 1992) and is not modulated by selective spatial attention (García-Larrea et al., 1991). In contrast, the later $\mathrm{N} 1$ waves of $\mathrm{A} \beta$ - and $\mathrm{A} \delta$-ERPs can be modulated by spatial attention (Legrain et al., 2002).

\section{Effect of stimulus repetition on vertex ERP responses}

The negative-positive VW is the largest component of the EEG response elicited by sudden sensory stimuli. Converging evidence indicates that stimuli of virtually all sensory modalities can elicit a VW, provided that they are salient enough (Liang et al., 2010). It is therefore not surprising that the VW elicited by auditory stimuli repeated at $1 \mathrm{~Hz}$ decays following a function similar to the one observed here for somatosensory stimuli (Fruhstorfer et al., 1970). Even when considering experimental observations that did not formally model the response habituation, the maximum decrease in VW amplitude consistently occurs at the first stimulus repetition, for auditory (Ritter et al., 1968; Fruhstorfer et al., 1970), somatosensory (Larsson, 1956; Fruhstorfer, 1971; lannetti et al., 2008; Wang et al., 2010; Valentini et al., 2011; Ronga et al., 2013), and visual stimuli (Courchesne et al., 1975; Wastell and Kleinman, 1980). The similarity of the decay of the VW elicited by $A \beta$ and $A \delta$ stimuli (Figs. 1, 3, 4) further supports the multimodal nature of the neural generators of these signals (Mouraux and lannetti, 2009). The mechanisms underlying such sharp reduction of response amplitude at the first stimulus repetition are likely to be similar across sensory systems.

Before discussing the contribution of the present results in elucidating the functional significance of the VW, it is important to highlight the empirical evidence that the observed response habituation is not due to neural refractoriness of afferent neurons or to fatigue of primary receptors. A previous study recorded ERPs elicited by pairs of nociceptive stimuli delivered at short intervals, which could be either identical or variable across the block (Wang et al., 2010). Only when the inter-stimulus interval was constant across the block, the VWs elicited by the second stimulus were reduced in amplitude. The peak amplitude of the VWs elicited by the second stimulus was instead as large as the VWs elicited by the first stimulus when the inter-stimulus interval was variable, indicating that neither neural refractoriness nor fatigue can easily explain the sharp response decay to stimulus repetition.

Furthermore, if the sharp response habituation at the first stimulus repetition was determined by fatigue of primary sensory receptors, we would have observed different decay profiles for stimuli delivered in varying versus constant spatial locations. Indeed, the VW elicited by contact heat stimuli at long and variable intervals (8-10 s) decays much faster if the second stimulus is delivered at the same spatial location of the first (Greffrath et al., 2007). Instead, we observed remarkably similar patterns of ERP decay for both A $\delta$ laser stimuli delivered at different spatial locations and $A \beta$ electrical stimuli delivered in the same skin region. Additionally, electrical stimuli activate directly the axons in the nerve trunk, bypassing the receptor, further ruling out receptor fatigue as explanation for the $A \beta$-ERP habituation. Receptor fatigue might still contribute to the slow decrease in ERP magnitude observed across dozens of stimulus repetitions of laser 
stimuli (Greffrath et al., 2007), but certainly not to the dramatic reduction of ERP amplitude we observed after one single stimulus repetition.

The physiological significance of the VW remains to be properly understood. However, there is evidence that this large electrocortical response reflects neural activities related to the detection of salient environmental events (Jasper and Sharpless, 1956; Mouraux and lannetti, 2009) and execution of defensive movements (Moayedi et al., 2015; Novembre et al., 2018). The detection of salient events relies on a hierarchical set of rules that consider both their probability of occurrence and their defining basic features (Legrain et al., 2002; Wang et al., 2010; Valentini et al., 2011; Ronga et al., 2013; Moayedi et al., 2016). The present results are informative with respect to this functional framework. Indeed, stimulus repetition did not abolish the VW elicited by either $A \beta$ or $A \delta$ stimuli, although it reduced its amplitude already after the first stimulus repetition. Therefore, even when stimulus saliency is reduced by contextual factors, there is a residual activity of the VW generators, only minimally reduced after the first few stimulus repetitions (Figs. 1, 3b,d). These findings point toward the existence of an obligatory VW activity triggered by any sudden and detectable change in the environment, even when contextual modulations minimize its behavioral relevance.

Extensive evidence from cell physiology indicates that neural habituation to repeated stimuli arises from alterations of synaptic excitability. Even the simple gill-withdrawal reflex in Aplysia dramatically habituates at the first stimulus repetition (Byrne et al., 1978), due to a decreased drive from the sensory neurons onto follower motor neurons (Castellucci et al., 1970; Carew and Kandel, 1973). The temporal profile of this short-term habituation follows a fast decay function (Carew and Kandel, 1973), strikingly similar to that observed in this and other studies on the habituation of electrocortical responses in humans (Fruhstorfer et al., 1970; Greffrath et al., 2007). These synaptic changes have been interpreted as a hallmark of learning, and are central to the ability of the nervous system to adapt to environmental events (Carew and Kandel, 1973). Interpreting the decay of neural responses as functionally relevant for learning is not in contradiction with attentional interpretations: stimuli that are learned and recognized are likely to require less attentional resources than novel stimuli, and stimuli that need to be learned are typically more salient.

\section{Conclusion}

In conclusion, our results provide a functional characterization of the decay of the different ERP components when identical somatosensory stimuli are repeated at 1 $\mathrm{Hz}$. Nociceptive and non-nociceptive stimuli elicit ERPs that are obligatorily contributed by both lateralized and vertex components, even when stimulus repetition minimizes stimulus relevance. This challenges the view that lateralized waves are not obligatorily elicited by nociceptive stimuli. Furthermore, both the lateralized and the VWs habituate to stimulus repetition following similar decay functions, which most possibly cannot be explained in terms of fatigue or adaptation of skin receptors.

\section{References}

Bancaud J, Bloch V, Paillard J (1953) Encephalography; a study of the potentials evoked in man on the level with the vertex. Rev Neurol (Paris) 89:399-418. Medline

Baumgärtner U, Cruccu G, lannetti GD, Treede RD (2005) Laser guns and hot plates. Pain 116:1-3. CrossRef Medline

Bromm B, Treede RD (1984) Nerve fibre discharges, cerebral potentials and sensations induced by $\mathrm{CO} 2$ laser stimulation. Hum Neurobiol 3:33-40. Medline

Byrne JH, Castellucci VF, Kandel ER (1978) Contribution of individual mechanoreceptor sensory neurons to defensive gill-withdrawal reflex in Aplysia. J Neurophysiol 41:418-431. CrossRef Medline

Carew TJ, Kandel ER (1973) Acquisition and retention of long-term habituation in Aplysia: correlation of behavioral and cellular processes. Science 182:1158-1160. Medline

Castellucci V, Pinsker H, Kupfermann I, Kandel ER (1970) Neuronal mechanisms of habituation and dishabituation of the gillwithdrawal reflex in Aplysia. Science 167:1745-1748. Medline

Courchesne E, Hillyard SA, Galambos R (1975) Stimulus novelty, task relevance and the visual evoked potential in man. Electroencephalogr Clin Neurophysiol 39:131-143. Medline

Cover TM, Thomas JA (2006) Elements of information theory, Ed 2. Hoboken, NJ: Wiley-Interscience.

Davis H, Osterhammel PA, Wier CC, Gjerdingen DB (1972) Slow vertex potentials: interactions among auditory, tactile, electric and visual stimuli. Electroencephalogr Clin Neurophysiol 33:537-545. Medline

Forss N, Narici L, Hari R (2001) Sustained activation of the human SI cortices by stimulus trains. Neuroimage 13:497-501. CrossRef Medline

Frot M, Rambaud L, Guénot M, Mauguière F (1999) Intracortical recordings of early pain-related $\mathrm{CO} 2$-laser evoked potentials in the human second somatosensory (SII) area. Clin Neurophysiol 110: 133-145. Medline

Frot M, Magnin M, Mauguière F, Garcia-Larrea L (2013) Cortical representation of pain in primary sensory-motor areas (S1/M1)-a study using intracortical recordings in humans. Hum Brain Mapp 34:2655-2668. CrossRef Medline

Fruhstorfer H, Järvilehto T, Soveri P (1969) Short-term habituation and dishabituation of the sensory evoked response in man. Acta Physiol Scand 76:14A-15A. Medline

Fruhstorfer H, Soveri P, Järvilehto T (1970) Short-term habituation of the auditory evoked response in man. Electroencephalogr Clin Neurophysiol 28:153-161. Medline

Fruhstorfer H (1971) Habituation and dishabituation of the human vertex response. Electroencephalogr Clin Neurophysiol 30:306312. Medline

García Larrea L, Bastuji H, Mauguière F (1992) Unmasking of cortical SEP components by changes in stimulus rate: a topographic study. Electroencephalogr Clin Neurophysiol 84:71-83. Medline

García-Larrea L, Bastuji H, Mauguière F (1991) Mapping study of somatosensory evoked potentials during selective spatial attention. Electroencephalogr Clin Neurophysiol 80:201-214. Medline

Garcia-Larrea L, Frot M, Valeriani M (2003) Brain generators of laser-evoked potentials: from dipoles to functional significance. Neurophysiol Clin 33:279-292. Medline

Golub GH, Reinsch C (1970) Singular value decomposition and least squares solutions. Numer Math 14:403. CrossRef

Greffrath W, Baumgärtner U, Treede RD (2007) Peripheral and central components of habituation of heat pain perception and evoked potentials in humans. Pain 132:301-311. CrossRef Medline

Hu L, Valentini E, Zhang ZG, Liang M, lannetti GD (2014) The primary somatosensory cortex contributes to the latest part of the cortical response elicited by nociceptive somatosensory stimuli in humans. Neuroimage 84:383-393. CrossRef Medline

Huang G, Xiao P, Hung YS, lannetti GD, Zhang ZG, Hu L (2013) A novel approach to predict subjective pain perception from singletrial laser-evoked potentials. Neuroimage 81:283-293. CrossRef Medline 
lannetti GD, Leandri M, Truini A, Zambreanu L, Cruccu G, Tracey I (2004) Adelta nociceptor response to laser stimuli: selective effect of stimulus duration on skin temperature, brain potentials and pain perception. Clin Neurophysiol 115:2629-2637. CrossRef Medline

lannetti GD, Hughes NP, Lee MC, Mouraux A (2008) Determinants of laser-evoked EEG responses: pain perception or stimulus saliency? J Neurophysiol 100:815-828. CrossRef Medline

Jasper H, Sharpless S (1956) Habituation of the arousal reaction. Brain 79:655-680. Medline

Jin QQ, Wu GQ, Peng WW, Xia XL, Hu L, lannetti GD (2018) Somatotopic representation of second pain in the primary somatosensory cortex of humans and rodents. J Neurosci 38:5538-5550. CrossRef Medline

Larsson LE (1956) The relation between the startle reaction and the non-specific EEG response to sudden stimuli with a discussion on the mechanism of arousal. Electroencephalogr Clin Neurophysiol 8:631-644. Medline

Lee MC, Mouraux A, lannetti GD (2009) Characterizing the cortical activity through which pain emerges from nociception. J Neurosci 29:7909-7916. CrossRef Medline

Legrain V, Guérit JM, Bruyer R, Plaghki L (2002) Attentional modulation of the nociceptive processing into the human brain: selective spatial attention, probability of stimulus occurrence, and target detection effects on laser evoked potentials. Pain 99:21-39. Medline

Liang M, Mouraux A, Chan V, Blakemore C, lannetti GD (2010) Functional characterisation of sensory ERPs using probabilistic ICA: effect of stimulus modality and stimulus location. Clin Neurophysiol 121:577-587. CrossRef Medline

Machens CK, Romo R, Brody CD (2010) Functional, but not anatomical, separation of "what" and "when" in prefrontal cortex. J Neurosci 30:350-360. CrossRef Medline

Mancini F, Bauleo A, Cole J, Lui F, Porro CA, Haggard P, lannetti GD (2014) Whole-body mapping of spatial acuity for pain and touch. Ann Neurol 75:917-924. CrossRef Medline

Mancini F, Beaumont AL, Hu L, Haggard P, lannetti GD (2015) Touch inhibits subcortical and cortical nociceptive responses. Pain 156: 1936-1944. CrossRef Medline

Maris E, Oostenveld R (2007) Nonparametric statistical testing of EEG- and MEG-data. J Neurosci Methods 164:177-190. CrossRef Medline

Moayedi M, Liang M, Sim AL, Hu L, Haggard P, lannetti GD (2015) Laser-evoked vertex potentials predict defensive motor actions. Cereb Cortex 25:4789-4798.

Moayedi M, Di Stefano G, Stubbs MT, Djeugam B, Liang M, lannetti GD (2016) Nociceptive-evoked potentials are sensitive to behaviorally relevant stimulus displacements in egocentric coordinates. eNeuro 3. CrossRef

Mountcastle VB (2005) Neural mechanisms of somatic sensation. Cambridge, MA: Harvard University Press.

Mouraux A, lannetti GD (2009) Nociceptive laser-evoked brain potentials do not reflect nociceptive-specific neural activity. J Neurophysiol 101:3258-3269. CrossRef

Mouraux A, lannetti GD, Plaghki L (2010) Low intensity intraepidermal electrical stimulation can activate $A \delta$-nociceptors selectively. Pain 150:199-207. CrossRef Medline

Mouraux A, De Paepe AL, Marot E, Plaghki L, lannetti GD, Legrain V (2013) Unmasking the obligatory components of nociceptive event-related brain potentials. J Neurophysiol 110:2312-2324. CrossRef Medline

Novembre G, Pawar VM, Bufacchi RJ, Kilintari M, Srinivasan M, Rothwell JC, Haggard P, lannetti GD (2018) Saliency detection as a reactive process: unexpected sensory events evoke corticomuscular coupling. J Neurosci 38:2385-2397. CrossRef Medline

Ohara S, Crone NE, Weiss N, Treede RD, Lenz FA (2004) Cutaneous painful laser stimuli evoke responses recorded directly from primary somatosensory cortex in awake humans. J Neurophysiol 91:2734-2746. CrossRef Medline

Peyron R, Frot M, Schneider F, Garcia-Larrea L, Mertens P, Barral FG, Sindou M, Laurent B, Mauguière $F$ (2002) Role of operculoinsular cortices in human pain processing: converging evidence from PET, fMRI, dipole modeling, and intracerebral recordings of evoked potentials. Neuroimage 17:1336-1346. Medline

Ritter W, Vaughan HG Jr, Costa LD (1968) Orienting and habituation to auditory stimuli: a study of short term changes in average evoked responses. Electroencephalogr Clin Neurophysiol 25:550556. CrossRef

Ronga I, Valentini E, Mouraux A, lannetti GD (2013) Novelty is not enough: laser-evoked potentials are determined by stimulus saliency, not absolute novelty. J Neurophysiol 109:692-701. CrossRef Medline

Sengupta AM, Mitra PP (1999) Distributions of singular values for some random matrices. Phys Rev E Stat Phys Plasmas Fluids Relat Interdiscip Topics 60:3389-3392. Medline

Snyder E, Hillyard SA (1976) Long-latency evoked potentials to irrelevant, deviant stimuli. Behav Biol 16:319-331. Medline

Spiegel J, Hansen C, Treede RD (1996) Laser-evoked potentials after painful hand and foot stimulation in humans: evidence for generation of the middle-latency component in the secondary somatosensory cortex. Neurosci Lett 216:179-182. Medline

Sutton S, Braren M, Zubin J, John ER (1965) Evoked-potential correlates of stimulus uncertainty. Science 150:1187-1188. Medline

Treede RD, Kief S, Hölzer T, Bromm B (1988) Late somatosensory evoked cerebral potentials in response to cutaneous heat stimuli. Electroencephalogr Clin Neurophysiol 70:429-441. Medline

Valentini E, Torta DM, Mouraux A, lannetti GD (2011) Dishabituation of laser-evoked EEG responses: dissecting the effect of certain and uncertain changes in stimulus modality. J Cogn Neurosci 23:2822-2837. CrossRef Medline

Valentini E, Hu L, Chakrabarti B, Hu Y, Aglioti SM, lannetti GD (2012) The primary somatosensory cortex largely contributes to the early part of the cortical response elicited by nociceptive stimuli. Neuroimage 59:1571-1581. CrossRef Medline

Valeriani M, Barba C, Le Pera D, Restuccia D, Colicchio G, Tonali P, Gagliardo O, Treede RD (2004) Different neuronal contribution to N20 somatosensory evoked potential and to CO2 laser evoked potentials: an intracerebral recording study. Clin Neurophysiol 115:211-216. Medline

Van Den Broeke EN, Mouraux A, Groneberg AH, Pfau DB, Treede RD, Klein T (2015) Characterizing pinprick evoked brain potentials before and after experimentally-induced secondary hyperalgesia. J Neurophysiol 114:2672-2681.

Venkatesan L, Barlow SM, Popescu M, Popescu A (2014) Integrated approach for studying adaptation mechanisms in the human somatosensory cortical network. Exp Brain Res 232:3545-3554. CrossRef Medline

Wang AL, Mouraux A, Liang M, lannetti GD (2010) Stimulus novelty, and not neural refractoriness, explains the repetition suppression of laser-evoked potentials. J Neurophysiol 104:2116-2124. CrossRef

Wastell DG, Kleinman D (1980) Fast habituation of the late components of the visual evoked potential in man. Physiol Behav 25:9397. Medline 